\title{
Gestational musculoskeletal disorders and corelated discomforts
}

\author{
Sabrina Santos Firmino ${ }^{1}$, Cássio Marcelo Cortez Fagá ${ }^{2}$, Vanessa Penha Basqueroto ${ }^{3}$, Aline do Nascimento Benitez ${ }^{4,5 *}$, Juliana Mendonça \\ Ferreira $^{6}$, Jessica Fernanda Barros Romano ${ }^{6}$, Catarina Villares Stoppe ${ }^{6}$, Gabriel Poi Junqueira de Oliveira ${ }^{7}$, Jancarlo Ferreira Gomes ${ }^{4,5}$ and $^{2}$ \\ Katia Denise Saraiva Bresciani ${ }^{7}$ \\ ${ }^{1}$ Araçatuba hippotherapy Center, Araçatuba, São Paulo, Brazil \\ ${ }^{2}$ São José do Rio Preto Children's Hospital and Maternity, São José do Rio Preto, São Paulo, Brazil \\ ${ }^{3}$ Gynecology and obstetrician Unisalesiano internship advisor, Araçatuba, São Paulo, Brazil \\ ${ }^{4}$ Faculty of Medical Sciences, University of Campinas [UNICAMP], Campinas, São Paulo, Brazil 5Laboratory of Image Data Science, Institute of Computing, \\ UNICAMP, Campinas, São Paulo, Brazil \\ ${ }^{6}$ São Paulo State University [Unesp], School of Veterinary Medicine, Araçatuba-São Paulo, 16050-680, Brazil \\ ${ }^{7}$ Unisalesiano University, Physiotherapy course, Araçatuva-São Paulo, 16016-500, Brazil
}

\begin{abstract}
The morphological and physiological transformations that occur during pregnancy involve hemodynamic, hormonal and biomechanical adaptations that can generate musculoskeletal disorders and reflect in various physical discomforts. Thus, in this research, the objective was to verify the main musculoskeletal alterations associated with the discomforts of pregnancy through a systematic review of the literature. The study was conducted from November of 2018 to February of 2019 , by the PICO strategy, with indexes "Physiotherapy" OR "Posture" AND "Discomfort" AND "Biomechanics" OR "Pregnancy" in PubMed, Scielo and Lilacs databases. After exclusion and inclusion criteria, 25/87 localized articles were selected for data extraction. The hemodynamic, hormonal and biomechanical changes of the pregnant woman reflect in the presence of oedema, increased body weight, ligament laxity and postural changes such as head and shoulder anteriority, hyperlordosis, pelvic anteversion, knee hyperextension, base enlargement, difficulty in the gait that lead to the appearance of musculoskeletal disorders, such as neck pain, thoracic duct syndrome, lower back pain, sacroiliac pain, plantar pressure and also the compromise of postural balance. The study of these disorders provides an indication of persistent pain symptoms during pregnancy and postpartum and knowledge of these changes helps to explain why they occur and the reason for the persistence of this sometimes even limiting pain in the pregnant woman.
\end{abstract}

\section{Introduction}

Pregnancy consists of a natural physiological process comprised of the sequence of adaptations occurring in the woman's body from fertilization 1 and is marked by 42 weeks that are divided into 3 stages. All women experience some musculoskeletal discomfort in the gestational period and the alterations that justify them in this period are: increased body weight, ligament laxity, uterine growth, breast enlargement, lumbar lordosis increase [1-3], pelvic anteversion [2,3] displacement of the balance [2-4] and changes in the base of support [2-4], which occur due to the adequacy, hormonal, biomechanical and skeletal muscle that the body passes to the foetus.

Hormones play an important role in pregnancy, they act both on the mother and on the foetus, ensuring the fetal life until its development. Among all the hormones that undergo changes during the gestational period, we can mention the main ones responsible for participating in musculoskeletal disorders and discomforts, such as relaxin, estrogen, progesterone, chorionic gonadotrophin, prolactin and lactogen.

Relaxin along with mechanical stress and increased load confer greater joint mobility and increased ligament laxity thus increasing the risk of injury $[5,6]$. Estrogen causes enlargement of the vascular system to the uterus, dilation of the external sex organs and the vaginal opening, relaxation of the pelvic ligaments that allow dilatation of the pelvic canal, which provides a greater pathway for the passage of the foetus and the rapid breast growth [5,6-8]. Progesterone during pregnancy becomes available for fetal use of additional amounts of nutrients stored in the endometrium [5,6], in addition, progesterone exerts a potent inhibitory effect on the uterine musculature, causing it to remain relaxed throughout the pregnancy $[5,6]$. Chorionic gonadotropin has the function of stimulating the secretion of testosterone by the fetal testis and helps in the development of the male foetus $[5,6,8]$. The hormone prolactin, produced by the placenta and is associated with another called placental lactogen, has the responsibility of leaving the mammary glands suitable for future milk production and helps to retain liquid [5].

Due to the appearance of these alterations, the musculoskeletal system generates corporal adaptations that provokes structural modifications in the static and dynamic skeletal system [1,9]. Body weight gain and protrusion of the abdomen cause an increase in lumbar

${ }^{\star}$ Correspondence to: Aline do Nascimento Benitez, School of Medical Sciences and Institute of Computing, University of Campinas, Campinas, São Paulo, Brazil, Tel: + +55 (18) 981993849; E-mail: benitez.alinenascimento@gmail.com

Key words: physiotherapy, posture, discomfort, biomechanics, pregnancy

Received: December 20, 2019; Accepted: January 13, 2020; Published: January 17,2020 
lordosis and alters the balance of the body, with muscle overload and fatigue $[1,10]$. The muscles of the abdominal wall may not be able to withstand the tension caused by weight gain, especially in the final stage of gestation and lose tonicity and strength [diastasis of the straight muscles] that in the postpartum can result in a more flabby belly [11]. Increased water retention may cause carpal tunnel syndrome, de Quervain's estonosantic tenosynovitis $[1,12]$. Pelvis ligaments are more demanding and become a source of pain, according to Souza, these changes become evident after the sixteenth week of gestation, when hyperextension of the knees, tension in the paravertebral muscles, swelling of the feet and decrease of the longitudinal arch of the feet are noticed $[2,3,13]$. The feet are slightly flattened, there is a drop in the medial plantar arch, and an increase in their length, and in some cases, it may even pronate $[3,6,14]$. The positions adopted, which combine to produce a tension in posture, are characteristic and include bent shoulders, bent elbows, adducted arms, interlocking or tight hands; head, trunk, ribs, knees and flexed ankles [15]. Maintaining this posture can overload some muscle groups and trigger pains $[1,9,10,15,16]$.

The changes in skeletal mechanics are due mainly to the hormonal action that increases ligament laxity [1,10,17-25], as well as biomechanical and musculoskeletal changes that cause structural changes in skeletal stability and dynamics especially gait adaptations $[1,26]$. The initialization of the gait is quite complex, since the body moves out of a "comfort" position to initiate the movement, which is called the first step [17]. This onset may be affected by weight gain which reduces walking speed and induces pelvic pain, changes in the feet dimensions of the pregnant woman, such as increase in length, width and volume, and reduction of plantar arch height $[6,14]$, causes discomfort for the pregnant woman $[1,3]$.

Each woman can present one or more changes, which can cause, pain and discomfort in daily activities, throughout the gestational period and may extend to the puerperium [18].

This study is justified considering the need to know the adjustments that occur in the body biomechanics during pregnancy, in order to minimize discomforts and pain, and to avoid possible losses such as fetal death $[1,3,19]$.

The main objective of this study is to relate musculoskeletal changes to the discomforts of pregnancy.

\section{Material and methods}

This systematic review of the literature was constructed through the steps of establishing the problem as a question; definition of the inclusion and exclusion criteria; sample selection; data extraction; identification of similarities and conflicts, presentation and discussion of findings and conclusion [27].

The problem was established by the guideline question "Are skeletal changes related to physical discomforts in pregnant women?", Elaborated according to the Patient, Intervention, Comparison and Outcomes [PICO] strategy [1,20]. All the studies published in the last 20 years, between 1998 and 2018, in English, and Portuguese in scientific article format, were included in the research. The databases used were PubMed, Scielo, and LILACS, with the descriptors "Physiotherapy OR Posture OR Biomechanics AND Discomfort AND Pregnancy". Duplicate publications in the databases were considered only once.

\section{Results}

In the search strategy, carried out on January 2, 2019, 87 articles were found among the different platforms, which after applying the exclusion criteria, followed by triage by title and reading of the abstract, were reduced to 25 publications for extraction of (Figure 1).

The alterations and associated discomforts were categorized according to the anatomical region in cervical, scapular waist, wrist and hand, thoracic spine, lumbar spine, pelvis and sacrum, knee, ankle and foot (Table 1).

\section{Discussion}

The articles selected for data extraction were published in the form of reflective or systematic reviews of the literature distributed between 1998 and 2018 and the results presented were distinct among the surveys, suggesting the absence of external factors that could have influenced. However, there was a preference for lumbar pain in pregnancy in $8 / 25$ publications where it was exclusively reported.

The inevitable changes in pregnancy are uncomfortable, but with a correct therapeutic institution they can be softened, which directed the objective of reporting in this work, all the changes of this period and their related discomforts.

During the first of three gestational trimesters, several physiological changes range from hormone levels to muscle skeletal adaptations, such as the recommended weight gain of 11 to $13 \mathrm{~kg}$, which lead to limiting discomforts reported by pregnant women as unbearable [21].

The hormonal increase that contributes to skeletal muscle discomforts and biomechanical adaptations during gestation are mainly related to relaxin, which promotes ligament laxity and is directly related to the action of two other hormones, progesterone and oestrogen and the first which reduces smooth muscle tone, increases body temperature by up to $0.5^{\circ} \mathrm{C}$, promotes the development of alveolar cells, milk-producing glands and production of fat deposits. While the latter promotes the growth of the uterus and ducts mammals and prepare the breasts for lactation, with higher levels of prolactin and lactogen.

The intense adaptation of the woman's body can be verified by the displacement and expansion of the uterus, a pelvic localization organ that can present up to six times its original size and requires a new clinical approach, such as an abdominal organ $[4,8,14]$. For the pregnant woman to maintain her postural stability, postural compensations

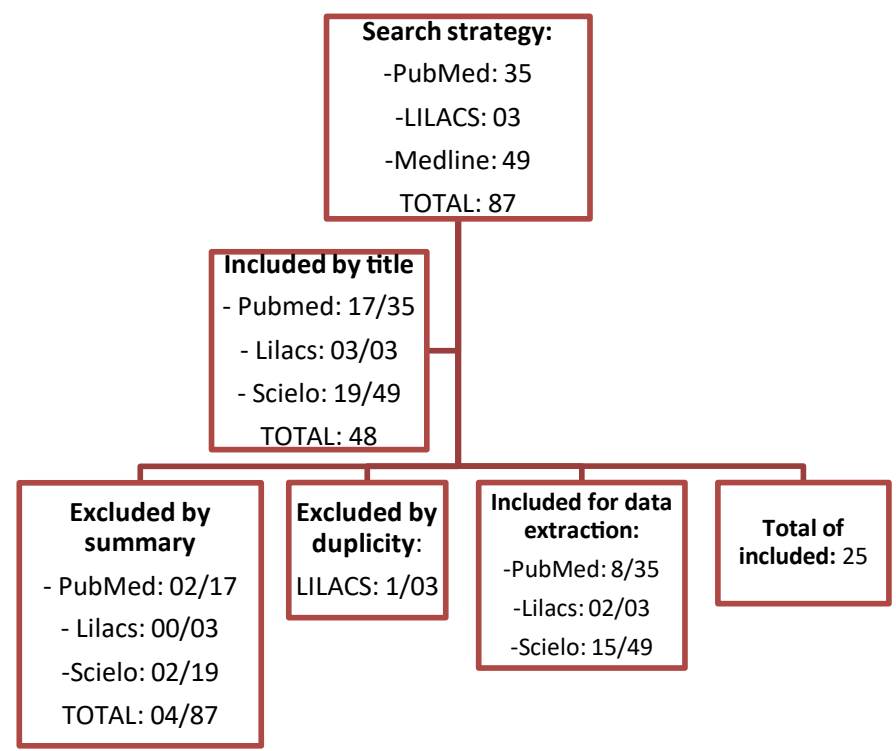

Figure 1. Search strategy flowchart 
Table 1. Skeletal muscle changes associated with gestational discomfort

\begin{tabular}{|c|c|c|c|}
\hline Region & Disorder & Discomfort & Gestational Age in Weeks \\
\hline Cervical & $\begin{array}{l}\text { Cervical hyperlordosis }[11,13] \\
\text { Head anteriorization }[6,10,11]\end{array}$ & $\begin{array}{l}\text { Decreased mobility; } \\
\text { Loss of visual field }[6] \\
\text { Loss of balance. }\end{array}$ & 27 a 41 \\
\hline Shoulder girdle & $\begin{array}{l}\text { Breast enlargement }[3,5,7,10,11,13] \\
\text { Muscle shortening; scapular stabilizers [12]; } \\
\text { Scapular waist becomes more rounded and arched [11, 12]; } \\
\text { Internal rotation of the upper limbs }[11,12] \\
\text { Scapula protraction [12]; } \\
\text { Thoracic hyperkyphosis [5]. }\end{array}$ & $\begin{array}{l}\text { Thoracic outlet syndrome [1]; } \\
\text { Loss of balance. }\end{array}$ & 0 a 13 \\
\hline Wrist and hand & Carpal tunnel syndrome $[1,19,21]$. & $\begin{array}{l}\text { Numbness or tingling of the thumb and the next two or three } \\
\text { fingers, one or both hands, and may extend to the elbow. }\end{array}$ & 4 a 26 \\
\hline Thoracic Spine & Thoracic hyperkyphosis [5]. & $\begin{array}{l}\text { Back pain; } \\
\text { Loss of balance. }\end{array}$ & 27 a 41 \\
\hline Lumbar spine & Lumbar hyperlordosis $[3,5,9,11,13]$. & $\begin{array}{l}\text { Low back pain }[3,8,9,11,16,18,19,20,21,24] \\
\text { Loss of balance. }\end{array}$ & 14 a 26 \\
\hline Pelvis and sacrum & $\begin{array}{l}\text { Pelvic anteversion }[3,6,11,14] \\
\text { Pubic symphysis diastasis [19]; } \\
\text { External rotation of hips. }\end{array}$ & $\begin{array}{l}\text { Posterior pelvic or sacroiliac pain; [1] } \\
\text { Loss of balance. }\end{array}$ & 14 a 26 \\
\hline knee & Hyperextension on the knees $[3,6,11,10,13]$. & $\begin{array}{l}\text { Femuropatellar syndrome; } \\
\text { Loss of balance. }\end{array}$ & 14 a 26 \\
\hline Ankle, foot & $\begin{array}{l}\text { Increased weight in the heel }[6,7,13] ; \\
\text { Extended support base }[5,6,7,11,13,16] ; \\
\text { Anserine gait }[10,11,16,21] ; \\
\text { Decreased plantar arch }[11]\end{array}$ & $\begin{array}{l}\text { Achilles pain }[6,7] \text {; } \\
\text { Ankle ininstability.oss of balance }\end{array}$ & 27 a 41 \\
\hline
\end{tabular}

will arise in response to the gravid uterus, enlargement of the breasts and body mass, which can move the centre of gravity up and forward $[4,10,13]$. During the gestational age the discomforts are intensified by the enlargement of the breasts and uterus and consequent displacement of the centre of gravity, in this way, there is an inevitable adjustment of the spine with the increase of the cervical and lumbar curvatures.

In response to the enlargement of breast volume and weight, cervical curvature can exacerbate and promote other changes such as adjusting the optical straightening reflex to keep the eyes in a horizontal plane, exacerbated shoulder protrusion that increases the workload of the muscles of the back of the neck to prevent the head from facing down. All these corrections are indispensable for the postural adaptation of the pregnant women, since without them the woman would lose her centre of gravity and fall as she walks $[4,10,13,20,22]$.

In smaller anatomical regions such as wrists and hands. There is a discomfort known as carpal tunnel syndrome, characterized by compression of the median nerve when passing through the narrow carpal tunnel under flexor retinaculum, and compression of structures and soft tissues due to marked oedema [22].

The enlarged breasts generate the shortening of the pectoral muscles and the reduction of the force of the stabilizing muscles of the scapula, accompanied by the scapular waist and the upper part of the spine that become more rounded and arched, causing an internal rotation of upper limbs and scapular protraction $[1,23]$.

With misalignment of the shoulder girdle, the high cervical spine tends to increase lordosis to compensate for alignment, leading to anteriority of the head, while the thoracic spine, to compensate for poor alignment of the shoulder girdle and cervical spine, increases its kyphosis, which can generate new discomforts. Thus, cervical hyperlordosis and anterior head can lead to decreased mobility of the region, loss of visual field and balance $[8,11,24]$. These changes, together with generalized oedema, cause a common compressive syndrome in situations of postural changes in the neck, fluid retention, hormonal changes or circulatory impairment called thoracic duct syndrome [23,25].
Martins e Silva et al. [10] carried out a study with 97 pregnant women evaluated by questionnaire and verified that $50 \%$ of the pregnant women reported pain in the spine at some period of gestation, with a higher occurrence of reports in the lumbar region and/or the sacroiliac. It was discussed that these discomforts would probably be related to the increase of the physiological curvatures such as thoracic kyphosis, which is considered a biomechanical alteration of the anatomical position of the pregnant woman that can be caused by pelvic anteversion $[11,20]$.

Lumbar pain also reported as posterior pelvic pain or sacroiliac pain, is a discomfort reported by pregnant women frequently $[1,4,8,10,11,13]$. The symptoms are related to a set of physical changes verified by the anteversion of the pelvis, ligament laxity, horizontalization of the sacrum, activation of the posterior muscles of the spine that compensate for the joint instability caused by this looseness and changes in the centre of gravity. The result of the combination of these physical changes with the postural changes and the muscular imbalance is some type of discomfort that occurs when carrying out daily activities of the pregnant woman and can be reported as a cutting pain in the buttocks $[1,2,4,8,14,24]$.

By changing the centre of gravity and changing the axes of force decomposition of the sacrum, the knees become hyperextended, transferring the entire body weight to the calcaneus. This alteration causes greater pressure on the hindfoot, the enlarged base, and the collapse of the plantar arch and the development of a valgus ankle, which is revealed by a pain in the calcaneus, from light intensity to inability to wander. Other alterations associated with this discomfort are the femur-patellar syndrome and gait alteration due to the corporal adaptations of the gestational period $[4,14]$.

The gait during pregnancy is recognized as typical and may be called an anserine gait or, popularly, as the gait of the duck $[1,3,6,17]$. The gait of the non-pregnant human being is divided into three phases, with shock of calcaneus, foot planing and impulsion. This conformation is altered in the gestational period due to weakness conditions of the pelvic girdle muscles, especially the gluteus medius muscle, with an increase in the support base and non-dissociation of the pelvic-scapular 
girdle. The gait transforms into one [12] for the other at each step, to shift the weight of the body, resembling a duck, and thus using the hips as the main booster of the gait, which loses its conformation in characteristic phases $[1,3,6,17]$.

The alteration in the gait of the pregnant woman is also influenced by changes in the neck, shoulders and eyes, which reflect in the displacement of the body weight to the calcaneus to re-establish the balance. However, this correction increases the relaxation of the abdominal muscles and provokes a tendency to walk like a "duck" leading to pains mainly in the lumbar spine $[6,17]$.

In general, the woman's body is adapted to the major changes of the gestational period, which are verified from the discovery of pregnancy to the puerperium, where they are involved in calcaneus until the optical adjustment with head anterioration [12]. The discomforts begin to occur according to each alteration, reaching its limiting phase in the third gestational trimester, moment in which all the corporal structures have already adapted for the reception and development of the foetus $[1,12]$.

\section{Conclusion}

The changes that happen in the woman's body are important and necessary for the full development of pregnancy, although they may compromise the quality of life for the woman experiencing the pregnancy. They are necessary for the best accommodation of the foetus during the gestational period, and the discomforts arising from these adaptations have a limited time and occur so that the woman does not suffer falls or is exclusively in bed without being able to carry out simple activities of day to day life.

Therefore, musculoskeletal changes are a strong indicator of the persistence and painful symptoms during pregnancy mainly after the first trimester. The acknowledgement of these changes makes the health professional have the capacity to elaborate preventive or diagnostic measures and the early treatment of the symptoms, which contributes to reduce the risks to the health of the pregnant woman.

\section{References}

1. Gazaneo MM (1998) Introduction. Rev Bras Physical Activity Health 3: 13-21.

2. Moreira LS, Rosa S, Andrade DS, Soares V, Avelar IS De, et al. (2011) Postural, balance and low back pain changes during pregnancy. Femina 39: 5

3. Si R, Eco G (2007) Analysis of Plantar Pressure and Postural Balance. Rev Bras Fisioter 11: 1-6.

4. Mann L, Kleinpaul J, Texeira C, Konopka C (2008) Brain-sparing effect: comparison of diagnostic indices. Rev Bras Ginecol Obstet 21: 99-105.

5. Neves C, Medina JL, Delgado JL (2007) Endocrine changes and immunomodulation in pregnancy. Arq Med 21: 175-182.

6. Albino MAS, Moccellin AS, Firmento B da S, Driusso P. Changes in gait propulsion force during pregnancy: changes in foot dimensions. Rev Bras Ginecol e Obs 33: 164-169.

7. Bung P (1999) Pregnancy and exercise. Gynakologe 32: 386-392.
8. Novaes FS, Shimo AKK, Lopes MHB de M (2006) Low back pain during pregnancy. Rev Lat Am Nursing 14: 620-624.

9. Difiore J (2000) The complete guide to good postnatal fitness. São Paulo Manole 4 $57-61$.

10. Martins RF, Silva JLP (2006) Prevalence of back pain during pregnancy. Rev Assoc Med Bras 51: 144-147.

11. Sebben V, Perussolo C, Guedes JM, Tourinho-Filho H (2001) Hydrotherapeutic treatment for low back pain in pregnant women. Rev Perspect 35: 167-175.

12. Coutinho E de C, Silva CB da, Chaves CMB, Nelas PAB, Parreira VBC, Amaral MO, et al. (2015) Pregnancy and childbirth: What changes in the lifestyle of women who become mothers? Rev da Esc Enferm da USP 48: 17-24.

13. Rett MT, de Araújo FR, Rocha I, da Silva RA (2012) Diastasis of rectus abdominis muscle immediately postpartum of primiparous and multiparous after vaginal delivery. Fisioter Pesq 19: 236-241.

14. Calegari SS, Konopka CK, Balestrin B, Hoffmann MS, Souza FS de, et al. (2012) Results of two treatment schemes for pyelonephritis during pregnancy and correlation with the outcome of pregnancy. Rev Bras Ginecol and Obs 34: 369-375.

15. Polden M, Mantle J (2002) Physiotherapy in Gynecology and Obstetrics. ( $1^{\text {st }}$ edn), Santos, São Paulo, Brazil.

16. Oliveira K De, Santos R, Jacovassi LV, Machado CK (2019) Performance of Physiotherapy in pain in upper limbs that affect women in the postpartum period as a consequence of postural changes and overload. FisioSale Araçatuba:1-10.

17. Andrade SR de S, Nora FG da SA, Avelar IS de, Soares V, Amaral WN do, et al (2012) Evaluation of gait initiation in low-risk pregnant women in the three gestational trimesters. Rev Bras Ginecol and Obs 34: 376-380.

18. Strapasson MR, Nedel MNB (2011) Immediate puerperium: unraveling the meaning of motherhood. Rev Gaúcha Enferm 31: 521-528.

19. Thomazinho F, Sardinha WE, Manoel J, Filho DDM, Motta F (2008) Arterial complications of thoracic outlet syndrome Arterial complications of thoracic outlet syndrome. Braz Vascular J 7: 150-154.

20. Tocoginecologia D (2006) Urinary Prevention In The Third Quarter D a Gest. Rev Assoc Med Bras 52: 153-156.

21. Homsi C, Ferreira J (2001) Low Back Pain in Pregnancy in the Perspective of the Biomedical Model : a Critical Repercussões Para a Saúde Da Mulher. Latino-Am Enferm 9: 95-100.

22. Dias de GAO, (2016) Prevalence of clinical symptoms and factors associated with the occurrence of carpal tunnel syndrome in pregnancy. UNESP Institutional Repository.

23. Barbosa RP (2012) Painful shoulder syndrome and the main pathologies that cause scapular waist dysfunction. Produzido por Opera House: 1-17.

24. Leite AC da NMT, Araújo KKBC (2012) Diastasis of the rectus abdominis in puerperal women and its relationship with obstetric variables. Physiotherapy in Mov 25: 389-397.

25. Marnach ML, Ramin KD, Ramsey PS, Song SW, Stensland JJ (2003) Characterization of the relationship between joint laxity and maternal hormones in pregnancy. Obstet Gynecol 1101: 331-335. [Crossref]

26. Rodacki CL, Fowler NE, Rodacki AL, Birch K (2003) Stature loss and recovery in pregnant women with and without low back pain. Arch Phys Med Rehabil 84: 507-512. [Crossref]

27. Whittemore R, Knafl K (2005) The Integrative Review: Updated Methodology. $J A d v$ Nurs 52: 546-553. [Crossref]

Copyright: (C2020 Firmino SS. This is an open-access article distributed under the terms of the Creative Commons Attribution License, which permits unrestricted use, distribution, and reproduction in any medium, provided the original author and source are credited. 